\title{
Escuchando la voz de la infancia en los procesos de cambio e investigación educativos. Aproximación al estudio de las transiciones entre las etapas de educación infantil y educación primaria
}

\author{
JAVIER ARGOS GONZÁLEZ \\ MARÍA PILAR EZQUERRA MUÑOZ \\ ANA CASTRO ZUBIZARRETA \\ Departamento de Educación, Universidad de Cantabria, España
}

\section{Introducción}

En los últimos años, se ha producido un incremento en el interés por escuchar la voz del niño y su perspectiva dentro del ámbito educativo. Se entiende esta participación activa como fruto de la nueva concepción existente sobre la infancia. Este nuevo concepto de infancia emergente, presenta al niño como un actor social de pleno derecho, en vez de un sujeto pasivo dentro de la sociedad. Esto implica que los niños sean considerados como individuos competentes, miembros fuertes y poderosos de la sociedad (Bruner, 1996; Dahlberg, Moss \& Pence, 1999) y como personas capaces, expertos en sus propias vidas y poseedores de conocimientos e intereses.

El ubicarnos en este nuevo marco provoca que la infancia comience a ser considerada digna de estudio al mismo nivel que se le venía concediendo a los estudios en los que participaban padres y educadores. IJames \& Prout, 1990; Corsaro \& Molinori, 2000; Christensen \& James 2000; Lloyd-Smith \& Tarr, 2000; O' Kane, 2000).

Escuchar la voz del niño en la investigación, amplía la visión que el investigador tiene de la realidad educativa, teniendo en cuenta que siempre ha sido una voz silenciada frente a la visión y perspectiva hegemónica de los agentes educativos adultos, tanto familiares como escolares.

Además, no podemos obviar que recoger la voz del niño supone reconocer, tal y como manifiestan Clark \& Moss (2001), que en muchas ocasiones los adultos tenemos una limitada comprensión de las vidas y experiencias que le afectan pues, tal y como señala Punch (2002), el adulto debe reconocer su incapacidad para participar plenamente en los mundos sociales e imaginarios de los niños, ya que nunca podrá regresar a la infancia.

Con el objetivo de disminuir el sesgo de la visión adulta, la Convención de los Derechos de la Infancia de Naciones Unidas de 1989, reconoce el derecho de la infancia a participar en las decisiones que afectan a su vida y a poder expresar su propio punto de vista. En los artículos 12 y 13 de la Convención, se destaca que el niño debe ser escuchado y respetado, lo que no significa • y no podemos olvidarlo• que se le otorgue el derecho a decidir o a anular la decisión de los demás. 
Aunque han pasado dos décadas desde la referida Convención, la existencia de estudios que recojan la visión o perspectiva infantil sigue siendo minoritaria. Consideramos que el aumento de estas investigaciones desde el campo social y educativo ayudará a cambiar la visión que se tiene de la infancia, posibilitando que se confiera al niño el papel de agente social, participante de pleno derecho y coinvestigador (James \& Prout, 1990; Christensen \& Prout, 2002; Bourdillon, 2004).

\section{Perspectivas en la investigación con niños: antecedentes y estado actual}

Tomando como referencia la aportación de Christensen \& Prout (2002), podemos distinguir cuatro perspectivas en la investigación efectuada con niños: el niño como objeto de investigación, el niño como sujeto, el niño como actor social y, finalmente, el niño como participante y co-investigador.

Si bien las dos primeras perspectivas son una constante a lo largo de la historia de la investigación social y educativa, las dos últimas responden a la reivindicación generada en las últimas décadas sobre el papel de la infancia en la sociedad. Nos encontramos ante perspectivas que coexisten en la actualidad, cuyo uso y grado de protagonismo depende de la percepción que el investigador tenga acerca de la infancia.

Las dos primeras perspectivas siguen siendo predominantes y suponen el tránsito desde la negación de la participación del niño en la investigación educativa, a diferentes niveles de participación que culminan con la participación plena en la que el niño se convierte en co-investigador.

La primera perspectiva, aquella que entiende al niño como objeto de investigación, es la más arraigada en la tradición investigadora. Supone negar la palabra al niño, que se convierte en objeto de estudio, una persona dirigida por el adulto en una sociedad que lo infravalora y destaca la dependencia infantil y su incompetencia. Para conocer al niño y generar conocimiento sobre la infancia se recoge información aportada por otros adultos que se convierten en referentes educativos para el niño: los padres y los maestros. Pero, también, existen investigaciones que no acuden a otros referentes educativos para obtener datos, sino que se dirigen a la fuente de origen, el niño, utilizando la observación de su conducta para, posteriormente, ser interpretada por el investigador.

Esta vertiente investigadora de larga tradición, que utiliza al niño como objeto de estudio, ha desarrollado la metodología observacional negando cualquier participación infantil y evitando, asimismo, la aparición del investigador en el contexto de estudio. Ver y no ser visto se convierte en una de las máximas del investigador.

Entre los estudios que podríamos referir como paradigmáticos de la perspectiva que concibe al niño como objeto en la investigación se encontrarían los desarrollados durante los años 60 por Bandura, con el experimento del muñeco bobo, que supuso la base del desarrollo de la teoría del aprendizaje social; los estudios observacionales de situaciones de separación del niño pequeño y su figura de apego, desarrollados por Bowlby, durante los años 50 y 60, que le condujeron a desarrollar la teoría del apego y pérdida y, avanzando tres décadas en el tiempo, las investigaciones de Schaffer que, a finales de los años 90, desarrolla los experimentos con niños sobre su resistencia a la tentación. 
Una característica común en los estudios citados es el papel protagonista conferido al investigador, que diseña las situaciones a observar realizando modificaciones a las que posteriormente el niño (sujeto pasivol es expuesto.

La segunda perspectiva se centra en el niño como un sujeto de investigación que tiene que ser tenido en cuenta, aunque su participación esté condicionada por la percepción y creencias que posea el investigador en relación con la capacidad cognitiva y competencias del pequeño. Esta perspectiva enfatiza el desarrollo madurativo del niño para su participación, así como el uso de la edad del niño como criterio para que éste pueda ser tenido en cuenta en la investigación educativa.

Un exponente de esta segunda perspectiva es Piaget que, durante los años 70, se aproxima a la infancia y a la riqueza del pensamiento infantil en un esfuerzo por recoger su voz directamente, a través del método clínico de interrogación al niño. Sus estudios suponen un cambio importante de perspectiva en las investigaciones sobre niños, dando comienzo al desempeño de un rol más activo por parte de la infancia.

Las dos últimas perspectivas intensifican el papel del niño en la investigación como un participante de pleno derecho, que se convierte en un agente social que tiene que ser escuchado en aquellos temas que le afecten y ser tenido en cuenta en la medida de lo posible, ya que será el primer beneficiario, y teniendo presente la sentencia defendida por algunos autores (Clark \& Moss, 2001; Lancaster \& Broadbent, 2003; Burke, 2005) de que "los niños son expertos en sus propias vidas". Es por ello, por lo que teniendo en cuenta el poder que se otorga a la infancia, cobra cada vez más fuerza la cuarta perspectiva, en la que el niño se convierte en co-investigador, consiguiendo una implicación y participación plena de la infancia en la investigación educativa.

Estas dos últimas perspectivas están muy cercanas a la pedagogía de la escucha desarrollada en Reggio Emilia, donde se establece un beneficio mutuo para niño y adulto, ya que tal y como expresa Rinaldi (2001):

Deberíamos escuchar a los niños, para que puedan expresar sus temores, pero también para que ellos nos den el valor de encarar los nuestros, por y con ellos; para que su sabiduría nos de confort, para que sus "por qué" orienten nuestra búsqueda por las razones y nos den la fuerza para encontrar respuestas no violentas, honestas y responsables; el coraje para el futuro y nos ayuden a encontrar una nueva manera de dialogar con ellos y con nosotros mismos.

En estos principios se basan las investigaciones desarrolladas por Clark \& Moss (2001) a través del método mosaico, que combina la metodología tradicional de observación y entrevista con la introducción de herramientas de participación con el objetivo de crear una imagen del mundo infantil, tanto individual como colectivo.

Pensamos que esta última perspectiva supone entender y asumir la exigencia ética del reconocimiento del otro, prestar atención a la palabra de los alumnos, ayudarles en orden a fundamentar esa palabra y a liberarles de las imposiciones restrictivas de su significado (Argos, Castro \& Ezquerra, 2009).

Si, como hemos constatado, parece de vital importancia garantizar que la infancia pueda expresar sus opiniones en aquellos asuntos que le afectan, es necesario encontrar métodos que nos ayuden a considerar su voz como elemento protagonista, como centro de la investigación. Así, ya en la década de los 
noventa surgen los métodos participativos como un mecanismo de reacción a la subordinación que, hasta entonces, tenía la infancia respecto del mundo adulto (Prout \& James, 1990).

Gallacher \& Gallagher (2008), señalan que la participación de los niños en la investigación educativa se convierte en un objetivo a lograr a la vez que en un método a seguir. Los métodos participativos pretenden dirigir cualquier pregunta de investigación directamente a los niños (Sayeed \& Guerin, 2000), desarrollándose para tal fin una variedad metodológica que intenta adaptarse, en la medida de lo posible, a la diversidad infantil. Entre los métodos más representativos podemos reseñar: el enfoque mosaico (Clark \& Moss, 2001), desarrollado en su estudio inicial, para "escuchar" a los niños pequeños ( 3 - 4 años de edad) y tener en cuenta sus opiniones y experiencias relacionadas con el funcionamiento de los servicios de atención a la primera infancia; el dibujo-conversación (Driessnack, 2006), utilizado en un estudio cualitativo con veintidós niños con edades comprendidas entre los siete y ocho años con el objeto de identificar y recoger los miedos infantiles más recurrentes; y finalmente, el uso de fotografias (Punch, 2002; Fasoli, 2003; Prosser, 2003), las conversaciones con niños (Mayall, 2000) y las hojas de trabajo (Punch, 2002), dirigidos a niños mayores de tres años. Este abanico de métodos participativos intenta, en la medida de lo posible, acercarse al niño desde su más tierna infancia de una forma lúdica, no invasiva y, sobre todo, familiar para él.

Hasta el momento se han realizado diferentes estudios que se han propuesto recoger la perspectiva infantil en relación con diferentes ámbitos o temáticas, ya sea mediante la utilización de alguno de los anteriores métodos, ya utilizando una combinación de ellos.

Por sólo referirnos a algunos focos en los que se ha centrado la reciente investigación con niños, podríamos apuntar los siguientes: las experiencias infantiles en la vida diaria, lla experiencia de los Consejos de Reggio Emilia: ver Del Río y otros, 2004), los roles del niño y del adulto y su participación y toma de decisiones en actividades (Miller,1997; Clark \& Moss, 2001), reglas, disciplina y resolución de conflictos (Miller, 1997; Finch, 1998), espacios de juego (Clark \& Moss, 2005) y transición educativa entre las etapas de Educación Infantil y Educación Primaria (Dockett \& Perry, 1999, 2005).

La existencia de estos estudios en la investigación educativa y el desarrollo de métodos participativos como los referidos, evidencian y apuntan la necesidad de incorporar cambios en los procesos de investigación educativa, fundamentalmente los relacionados con la escucha y con la participación de la infancia en los temas que la afectan.

Presentamos a continuación una tabla en la que plasmamos, de forma sintética, las anteriores perspectivas analizadas en la investigación con niños. En relación con cada una de ellas, se apuntan algunas diferencias que presentan en relación con una serie de parámetros relevantes. 
TABLA 1

\begin{tabular}{|c|c|c|c|}
\hline \multirow{2}{*}{$\begin{array}{l}\text { PARÁMETROS } \\
\text { DE CONTRASTE }\end{array}$} & \multicolumn{3}{|c|}{ PERSPECTIVAS EN LA INVESTIGACIÓN CON NIÑOS } \\
\hline & El niño como objeto & El niño como sujeto & $\begin{array}{l}\text { El niño como agente social. } \\
\text { El niño como co-investigador }\end{array}$ \\
\hline Concepto de niño & $\begin{array}{l}\text { Sujeto pasivo, incapaz de } \\
\text { participar. Dependiente. } \\
\text { Persona incapaz de detectar } \\
\text { sus propias necesidades. }\end{array}$ & $\begin{array}{l}\text { Sujeto activo condicionado } \\
\text { por su desarrollo } \\
\text { madurativo y su capacidad. } \\
\text { Persona incapaz de detectar } \\
\text { sus propias necesidades. }\end{array}$ & $\begin{array}{l}\text { Sujeto activo y de derecho, } \\
\text { autor de su propia vida. } \\
\text { "Experto" en aquellos temas } \\
\text { que le afectan. }\end{array}$ \\
\hline Propósito principal & $\begin{array}{l}\text { Conocer y proteger a la } \\
\text { infancia. }\end{array}$ & $\begin{array}{l}\text { Conocer y proteger a la } \\
\text { infancia. Esperar a la } \\
\text { maduración del niño para } \\
\text { ser tenido en cuenta. }\end{array}$ & $\begin{array}{l}\text { Ejercitar los propios derechos } \\
\text { de la infancia. Tener al niño en } \\
\text { cuenta con independencia del } \\
\text { criterio edad. }\end{array}$ \\
\hline $\begin{array}{l}\text { Roles y actitudes del } \\
\text { investigador ante el niño }\end{array}$ & $\begin{array}{l}\text { Investigador principal. } \\
\text { Paternalismo. } \\
\text { Directividad. }\end{array}$ & $\begin{array}{l}\text { Investigador principal. } \\
\text { Paternalismo. } \\
\text { Directividad. }\end{array}$ & $\begin{array}{l}\text { Co-investigador. } \\
\text { Escucha activa. }\end{array}$ \\
\hline Técnicas utilizadas & Observación. & $\begin{array}{l}\text { Observación. } \\
\text { Entrevista. }\end{array}$ & Técnicas participativas. \\
\hline
\end{tabular}

\section{Desafíos en la investigación con niños}

Tener en cuenta y recoger la voz de la infancia en aquellos temas que la afectan no es una tarea sencilla. De una parte, supone un reto individual por parte del investigador para identificar las necesidades y demandas de la infancia y, de otra, se conforma como un reto social a la hora de asumir que los niños son ciudadanos de pleno derecho de la comunidad en la que viven y como tales, deben participar y ser tenidos en cuenta como cualquier otro miembro de la sociedad.

Para poder afrontar y responder adecuadamente a esta dificil empresa, es necesario atender y reflexionar sobre algunos de los desafíos que se nos plantean cuando pretendemos llevar a cabo una investigación con niños.

\subsection{Mitigación de la disparidad de poder y de estatus entre adultos y niños}

El primer desafío consistiría en eliminar, en la medida de lo posible, la disparidad de poder y de estatus entre adultos y niños. Este aspecto supone tener muy presente el concepto de "simetría ética" propuesto por Christensen \& Prout (2002), que deberá ser tenida en cuenta en la tarea investigadora.

En relación con ello, uno de los valores que deberían guiar la investigación con niños sería aquel relacionado con la importancia de la interdependencia. Frente a las actitudes y conductas paternalistas y proteccionistas adultas que tienden a excluir a la infancia de muchos actos participativos, se nos plantea la necesidad de efectuar una valoración positiva del rol social que ha de jugar ésta y, en consecuencia, de escuchar y recoger su visión o perspectiva acerca de la realidad que viven. 
Desde este enfoque, el objeto de estudio (la infancia) y el investigador, dependen uno de otro, determinando el devenir de la investigación y el resultado final de la misma: se trataría de desarrollar una investigación con niños y no una investigación sobreniños.

En definitiva, supone resaltar que sólo podremos crear un espacio que permita a los niños hablar y ser escuchados cuando se rompa el desequilibrio de poder existente entre el adulto y el niño. Para favorecer el equilibrio deseado, el diálogo con los pequeños durante el proceso de investigación se convierte en una garantía para mantener el marco o perspectiva de investigación a la que nos queremos adscribir.

\subsection{Consideración de los condicionantes éticos}

De este primer desafío, ligado a la dimensión axiológica de la investigación, surge un segundo reto que nos demandaría atender a los condicionantes éticos de los trabajos con niños.

No podemos obviar que la investigación educativa no centra su estudio en realidades físicas o en objetos, sino que lo hace sobre acciones, ideas o pensamientos de personas y, por ello, hemos de ser conscientes del compromiso que adquirimos con ellas cuando pretendemos acercarnos a conocer mejor sus realidades. Y este compromiso, esta responsabilidad se agudiza, si cabe, cuando desarrollamos investigaciones con niños.

Este segundo compromiso lo podríamos formular como la necesidad de encontrar un equilibrio entre el derecho a la participación del niño y la dimensión ética. En relación con este propósito surgen dilemas, preguntas, que no ofrecen una solución única.

El gran peligro ético, para el investigador educativo, es dejar de hacerse preguntas en relación con al menos, tres ámbitos: los propósitos del trabajo, el proceso o desarrollo de esta investigación y las relaciones que se establecen con las personas involucradas en ella. Todas las preguntas y dilemas que surjan de estos ámbitos, están impregnadas de valores que han de guiar y sustentar la investigación educativa.

Los aspectos éticos clave a considerar en la investigación con niños han sido abordados primero por Alderson (1995) y posteriormente por Roberts (2000), quien, a partir del trabajo de la investigadora citada con anterioridad, realiza un intento de plantear interrogantes sobre los que reflexionar más que de escribir un determinado código ético. Su aportación se sintetiza en diez preguntas que deben acompañar al diseño y al desarrollo de la investigación con niños. Del conjunto de consideraciones apuntadas y, en un afán de síntesis, reflejamos a continuación las principales dimensiones que, a nuestro entender, deberían de considerarse en una investigación con niños. 
FIGURA 1

Dimensiones a considerar en la investigación con niños

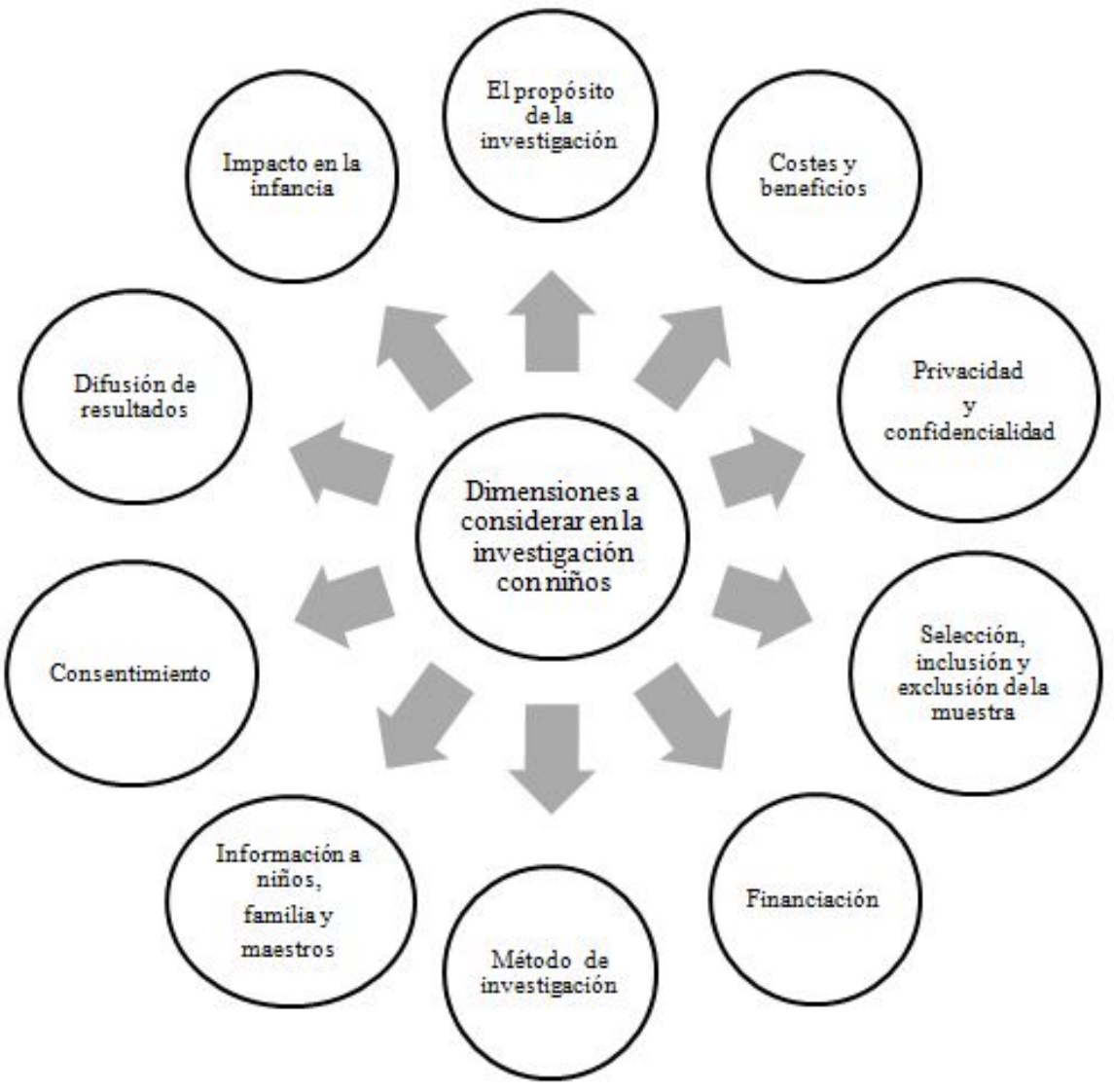

En definitiva, el investigador debe de tener siempre muy presente el bienestar de las personas objeto de estudio ya que no podemos olvidar que, a diferencia de lo que ocurre en otros ámbitos heurísticos, en la investigación educativa trabajamos con personas y éstas han de ser determinantes en el curso de nuestro estudio.

\subsection{Identificación y/o creación de métodos que recojan la voz de la infancia}

Si queremos favorecer el bienestar de las personas que participan en la investigación, un aspecto a cuidar es el método a utilizar. Con demasiada frecuencia se espera que los niños puedan adaptarse a los métodos de investigación utilizados con personas adultas, sin ser conscientes que lo que verdaderamente se necesita es un cambio institucional y organizativo que aliente y facilite la voz de los niños (Prout, 2002).

Con independencia de la incertidumbre que ello pueda generar, es necesario dirigir la pregunta de investigación directamente a los niños (Sayeed \& Guerin, 2000). Desde este planteamiento es desde donde podemos apuntar el tercer desafío: identificar o crear métodos con los que el niño pueda expresarse libre y fácilmente.

A pesar de haber mostrado anteriormente los principales métodos utilizados en la investigación actual sobre niños, éstos aún son minoritarios y escasos, por lo que necesitamos contar con un abanico más amplio que posibilite un acercamiento más natural a la infancia. 
En la creación de nuevos métodos consideramos imprescindible valorar la claridad del lenguaje a utilizar, los espacios y tiempos en los que se va a implementar, la familiaridad del método para el niño, el tipo de relación adulto-niño que permite y la posibilidad que proporciona de generar y analizar datos.

La conjunción de estas valoraciones hace que el investigador afronte el desafío de la innovación, entendida no sólo como una respuesta para adaptar los métodos a la infancia sino también como un proceso de mejora de lo ya existente en aras de potenciar su eficiencia. Es necesario tener en cuenta que toda experiencia que implique una innovación se ve afectada por un conjunto de obstáculos y dificultades que la afectarán. Su identificación, el modo en el que se afronten y la persistencia posibilitarán el éxito en la actividad que nos propongamos.

Algunos de estos obstáculos que identificamos son los siguientes:

- El peso de la tradición: la seguridad de lo conocido frente a la incertidumbre de lo nuevo.

- La lucha por la especialización: el dominio de un método frente al conocimiento y experimentación de nuevas técnicas.

- La validez de los resultados: la aprobación oficial y académica de los resultados con métodos tradicionales frente a una investigación con métodos innovadores recientes y por descubrir.

Es por ello por lo que la creatividad, la efectividad y el dinamismo son cualidades que todo grupo de investigación que afronte el estudio con niños debería poseer.

Un aspecto a destacar en el análisis de los métodos anteriormente apuntados y que han llevado a cabo investigación con niños, es la ausencia del uso de las TIC como recurso de investigación a pesar de que, actualmente, los niños asumen con total normalidad la presencia de las tecnologías en la sociedad en la que viven, conviviendo con ellas y adoptándolas sin dificultad para su uso cotidiano. Por ello, un desafío dependiente de la adaptación metodológica en la investigación con niños, es el uso de las TIC como medio para recoger datos que nos ayuden a conocer la visión de los pequeños. La competencia de la población infantil en el uso de medios tecnológicos y su fascinación por los mismos provoca que se conviertan en un recurso a tener en cuenta en la investigación educativa. Además, la versatilidad de estos medios parar adaptarse a las necesidades de los niños con algún tipo de necesidad educativa especial, los convierten en un método inclusivo frente a las limitaciones que pueden caracterizar a algunos de los restantes métodos expuestos.

La creación de algún método de investigación con niños, sustentado en el uso de medios tecnológicos como el ordenador o la pizarra digital, se puede convertir en una vía de futuro desarrollo en la búsqueda de métodos que faciliten la expresión libre, amena y sencilla por parte de la población infantil.

\subsection{Necesidad del consentimiento informado y de la reducción del poder adulto en la investigación educativa}

Si en la creación y definición del marco que guiará la investigación con niños encontramos elementos de tensión y desafíos a los que responder, en los inicios de la implementación de la investigación, el consentimiento informado supone un nuevo desafío, a la vez que un prerrequisito, para el investigador. El 
consentimiento informado ha sido objeto de debate a lo largo de los últimos años (Christensen \& Prout, 2002; Punch, 2002, Cocks, 2006) como consecuencia del rol activo asumido por la infancia en este tipo de investigación.

Hacer partícipe al niño de la investigación supone implicarle, desde el momento en el que se solicita su participación. Así, será el propio niño quien decida, a pesar del previo consentimiento familiar, su aceptación a participar en las actividades que se le propongan.

En el consentimiento informado podemos diferenciar con Cocks (2006) tres momentos claves: la presentación de la información, su comprensión por parte del niño y la emisión de una respuesta al respecto. La adecuación y adaptación de la información que se presente a las características del receptor posibilitará la emisión de una respuesta ajustada por parte del niño atendiendo a sus preferencias y respetando así su disposición hacia la actividad.

El último desafío que queremos aquí apuntar sería el de reducir el sesgo de los estudios que recogen en exclusividad la visión adulta. Pensar que el adulto conoce las necesidades, intereses y demandas del niño es erróneo, ya que los estudios muestran que la visión del adulto y del niño sobre realidades educativas que experimenta, no tienen por qué coincidir (Pramling, 2004).

Considerar este hecho nos ha de llevar a asumir que el conocimiento acerca de los niños será incompleto si no se tienen en cuenta los conocimientos que los propios niños tienen acerca de sí mismos. No se trata de rechazar la visión adulta sino, más bien, de complementarla realizando una triangulación de perspectivas: la del niño, la del maestro y la de los padres, en aquellos temas educativos objeto de investigación

\subsection{El investigador como agente de cambio social: sus creencias y prácticas en la investigación educativa}

Como venimos exponiendo, la perspectiva de investigación con niños supone una propuesta que recoge la visión y voz de la infancia de primera mano, una huida del adultocentrismo y el reconocimiento del poder y capacidad del niño para ser escuchado y tenido en cuenta. Supone por tanto, una revolución en el concepto tradicional de investigación que sólo puede plantearse o llevarse a cabo con el apoyo del investigador.

Es por ello por lo que la figura que puede asumir el investigador en este tipo de investigación ha de vincularse con su rol de agente de cambio social que potencia el protagonismo de la infancia en la investigación, desechando aquella otra que le entiende como un reproductor del modelo social existente que concibe la infancia como un colectivo subordinado al pensamiento e ideario adultos.

Será, por tanto, el pensamiento sostenido por el investigador el que le convierta, bien en un facilitador, bien en un limitador en la investigación con niños. La forma en la que los investigadores perciben la infancia influirá en el papel que el niño tenga en la sociedad y, más específicamente, en el rol que se le permita ostentar en la investigación educativa.

El estudio llevado a cabo recientemente por Powell \& Smith (2009) en Nueva Zelanda, es el primer acercamiento realizado al pensamiento del investigador y a la visión que tiene sobre el derecho de la 
participación de los niños en la investigación educativa. En él, se recogió, por medio de entrevistas, la opinión de doce investigadores de diferentes áreas de estudio que se encontraban inmersos en investigaciones con niños. A pesar de que sus investigaciones se enmarcaban en el enfoque participativo, lo cierto es que las conclusiones del mismo ofrecen un resultado contradictorio ya que, sus prácticas en lo referido al papel otorgado al niño, se acercan más a investigaciones que consideran al niño como objeto pasivo de estudio durante el proceso investigador, que a principios y actuaciones característicos de una investigación participativa.

Esta inconsistencia entre pensamiento y acción prueba lo difícil que resulta ajustar el comportamiento a las propias creencias cuando ello exige cambiar hábitos establecidos en cualquier dimensión de la vida de una persona, en este caso, en la investigación educativa.

Consideramos que la existencia de inconsistencias, a pesar de mostrar dificultades en la implementación de unas creencias condicionadas por el entorno social, supone un primer intento de cambio de pensamiento individual por parte del investigador ante el objeto de su estudio, un primer paso para lograr que el investigador educativo sea capaz de hacer oír la voz de la infancia. En definitiva, el inicio de un camino que lleve a ésta a adquirir el papel de agente de cambio social.

\section{La transición educativa entre el último curso de educación infantil y la educación primaria, desde la perspectiva infantil: estudio exploratorio}

\subsection{Propósitos y supuestos de la investigación}

Nuestro propósito es efectuar un acercamiento al estudio de la transición educativa entre las etapas de Educación Infantil y de Educación Primaria, tratando de recoger la mirada, de escuchar la voz de los pequeños que son, en definitiva, los verdaderos protagonistas de estos procesos de transición.

Pensamos que la participación de los niños en el estudio puede ampliar la visión que el investigador tiene de esta problemática pedagógica, posibilitando, de este modo, una mejora de los procesos de transición entre las etapas educativas anteriormente referidas.

Esperamos que, a raíz de esta experiencia, podamos generar en un futuro buenas prácticas que recojan las aportaciones de todos los implicados en este proceso de transición.

\subsection{Características del estudio: aspectos metodológicos \\ Diseño y Método}

Para tal fin, hemos iniciado en dos centros educativos un estudio piloto de carácter exploratorio, utilizando el método del dibujo-conversación. Cada uno de ellos cuenta con dos unidades destinadas a niños de cinco años. El primer centro, al que llamaremos A, tiene ubicadas las unidades de Educación Infantil y de Educación Primaria en dos edificios diferenciados, mientras que el segundo, al que a partir de ahora denominaremos $\mathrm{B}$, ubica las unidades de ambas etapas en el mismo edificio aunque en diferente piso. 
Seleccionamos el método del dibujo-conversación ya que consideramos el beneficio que podría entrañar el combinar dos métodos que entendemos como complementarios y que pueden ayudarnos a potenciar la participación infantil en un tipo de investigación como la que nos ocupa.

Partimos de las conversaciones con niños, en las que los pequeños apelan a sus estrategias comunicativas construyendo significados en colaboración, donde las comprensiones individuales y colectivas se vuelven más complejas gracias a las contribuciones sucesivas de las diferentes personas (Borzone y Rosemberg, 2000). Es un método rico pero complejo para el adulto ya que debe de ser capaz de no protagonizar ni dirigir las conversaciones con los niños, conversaciones que proporcionan información amplia y diversa a partir de un tema propuesto sobre el que hablar.

El hecho de reconocer que los niños saben mucho más de lo que sus respuestas orales nos sugieren hace que combinemos este método con el del dibujo, utilizado como medio para alentar a los niños a comunicarse de manera efectiva, sin la fuerte dependencia de las habilidades verbales y de alfabetización (Young y Barrett, 2001; Dockett y Perry, 2005), y como medio para ayudar a los niños a "hacer visibles sus pensamientos a los demás", a expresar sus experiencias, pensamientos, sentimientos y opiniones.(Robertson, 2000; Dockett y Perry, 2005). Un aspecto a destacar de este método es que el niño realiza una actividad conocida para él y que ha desarrollado con anterioridad tanto en el ámbito familiar como en el escolar.

\section{Procedimiento}

Al ser un elemento fundamental en este tipo de investigación el que los niños conozcan al adulto investigador y se sientan cómodos y seguros con él, éste es presentado en la asamblea de clase, participando activamente. Así, en esta asamblea se les comenta a los pequeños que le gustaría hablar con ellos y que le hicieran un dibujo y, a continuación, se les pregunta que quiénes de ellos querrían participar. Por tanto, la muestra de niños está conformada únicamente por pequeños que se han mostrado voluntarios a participar en las referidas actividades.

Con la finalidad de mitigar al máximo la posible inseguridad inicial que puedan tener los niños ante el adulto, las conversaciones se realizan con parejas de niños, llegándose a crear diálogos en los que el adulto apenas participa.

La sesión se inicia con una conversación que gira en torno a sus gustos y preferencias en Educación Infantil, a la ubicación del aula del curso que viene, a sus motivaciones y expectativas ante el cambio de etapa, a la información que poseen y quién se la ofrece y respecto de lo que piensan que se les va a demandar en la Educación Primaria. Esta información se complementa con la petición, por cierto muy bien acogida, de hacer un dibujo. Para ello, les entregamos una hoja dividida en dos mitades mediante una línea vertical. En un lado del folio se les pide que dibujen cómo es para ellos la escuela de ahora lla de Educación Infantill y en qué actividades les gusta participar mientras que, en la otra parte de la hoja deben dibujar cómo creen que es el colegio de Educación Primaria. En el caso del centro escolar B, que ubica las unidades de ambas etapas en el mismo edificio, preguntamos por cómo creen ellos que serán las clases del piso de arriba.

A lo largo de toda la sesión efectuamos grabaciones no sólo de las conversaciones sino, también, de los comentarios que los pequeños efectúan mientras realizan el dibujo. Estas últimas ayudan a los 
adultos a comprender las ideas que mantienen sobre lo dibujado, proporcionando información sobre los intereses de los niños y su trasfondo (Holliday et al, 2009).

\subsection{Avance de resultados}

De los datos obtenidos hasta este momento y generados tanto en las conversaciones de y con los pequeños como del análisis de sus dibujos, podríamos apuntar una serie de cuestiones o tópicos recurrentes entre los que destacamos los siguientes:

\section{Relaciones sociales y afectivas}

- La preocupación por hacer amigos y por mantener los ya existentes ante el tránsito de la etapa de Educación Infantil a la etapa de Educación Primaria, se convierte en una constante en todas las conversaciones. Ante el inicio del curso escolar en Primaria, se realiza una reorganización de los grupos-clase por lo que los niños muestran su preocupación por saber si van a estar con sus amigos o si, por el contrario, les van a separar. Esta preocupación aumenta al no recibir respuesta por parte del adulto a pesar de que han sido los referentes adultos, padres y maestros, los que han comunicado al pequeño el cambio de agrupamientos. Una práctica que se realiza en uno de los centros escolares consiste en preguntar a los niños con qué tres amigos desearían estar el curso próximo. Este hecho disminuye la ansiedad del niño, ya que a pesar de reestructurar los grupos, el pequeño se asegura el seguir manteniendo sus vínculos de amistad, a la vez que percibe la posibilidad de crear amistades nuevas con compañeros que se incorporen el curso que viene al colegio.

- La importancia de las relaciones sociales y afectivas para el niño se manifiesta de la misma forma en el dibujo, apareciendo figuras infantiles que representan al autor del dibujo junto a su amigo realizando diferentes actividades conjuntamente. Otro aspecto a destacar en los dibujos de los niños del centro A y que no ocurre en los dibujos de los alumnos del otro centro escolar, es la aparición de su maestro actual en el dibujo del centro de Educación Primaria, ya sea como intento de manifestar su deseo de continuidad, ya como la representación de esta figura conocida frente al desconocimiento del próximo referente educativo.

\section{Normas y conducta escolar}

Los niños conocen y expresan claramente las normas del centro de Educación Infantil, si bien creen que en el centro de Educación Primaria existirán normas que exigirán un mayor compromiso por su parte, aunque no sean capaces de verbalizarlas. De la misma forma, en las conversaciones se les pide que, desde su experiencia en la Educación Infantil, den algún consejo a aquellos niños que se incorporan el curso que viene a las aulas de tres años.

El hecho de que muchos niños tengan hermanos pequeños nos ayuda a centrar la pregunta y a que el niño la conteste con mayor entusiasmo. La mayoría de las respuestas giran en torno a la necesidad de conocer y "asumir" las reglas de comportamiento instauradas en el ámbito escolar. Su representación en el dibujo es escasa, salvo en el caso de aquellos pequeños que representan niños en fila a la salida de la clase o de los que dibujan niños en pupitres y verbalizan que en clase tienen que estar en silencio. 


\section{Conocimiento del espacio}

Desenvolverse en un entorno desconocido para el niño es uno de los retos a los que se enfrenta el alumno ante su transición educativa. Los pequeños centran su atención en las zonas de juego y expresan la importancia de conocer los espacios comunes y sobre todo, aquellos que les faciliten su autonomía como aseos, aulas... Así, en el centro A, los niños de Educación Infantil destacan el tamaño del edificio del centro de Educación Primaria. Este hecho es plasmado de forma recurrente en sus dibujos en los que presentan un edificio de grandes dimensiones repleto de ventanas. A pesar de que estos niños realizan algunas actividades en el centro de Educación Primaria, ninguno de sus dibujos representan los espacios interiores del centro de Educación Primaria, centrándose en la recreación del edificio y de las zonas de juego. Por su parte, los niños del centro B, que comparten edificio, optan por dibujarse a ellos mismos frente a un pupitre escribiendo, reconocen conocer las aulas de Primaria porque en alguna ocasión se les ha pedido hacer un recado, pedir material o llevarlo a esa clase. Priorizan esta experiencia como medio de conocimiento de la etapa superior frente a actividades conjuntas que se realizan entre etapas, tal vez por la autonomía que experimentan al descubrir por sí mismos otros espacios.

\section{Habilidades y destrezas académicas}

La iniciación en la lectura y escritura así como pequeños contenidos de aritmética son considerados por los niños como contenidos a tener en cuenta para considerarse preparados para entrar en la etapa de Educación Primaria. Así, algunos de ellos se comparan con hermanos mayores y, en concreto, en el conocimiento que, sobre lectura y escritura tenían éstos a su edad. El saber más que ellos les hace sentirse capacitados y seguros para pasar a la etapa de Primaria. A su vez, ya en Educación Infantil comienzan a exteriorizarse las diferencias percibidas por los propios niños en relación a su rendimiento escolar, llegando a identificar en conversaciones espontáneas a aquellos compañeros más avanzados y a aquellos otros que piensan que van a tener dificultades el curso que viene.

\section{El rol del alumno}

El mayor cambio percibido en el niño dentro de esta transición es en la metodología didáctica y en la asunción de un rol como alumno que responda a las demandas del docente. La aparición de los deberes, la disminución de los periodos de juego y la organización y disposición del aula aparecen como elementos recurrentes en las conversaciones.

Una constante del pensamiento del niño, recogida a través de las conversaciones, es su preocupación por el aprendizaje y rendimiento dentro de la etapa de Educación Primaria. Surge la visión de un "alumno competente" por parte del niño, inspirado en lo que es para él un alumno ideal: aquél capaz de lograr la adquisición de los aprendizajes instrumentales básicos y de responder a las demandas del docente.

\subsection{Apuntando algunas conclusiones para poder seguir avanzando en el proceso de escucha de la infancia}

A través del presente estudio exploratorio hemos conferido al niño el rol de sujeto activo y de pleno derecho, capaz de realizar aportaciones en todos aquellos temas que le afectan, percibiéndole como persona experta que experimenta en primera persona la transición educativa. 
El estudio pone de manifiesto que el niño debe ser tenido en cuenta, con independencia del criterio edad, si conseguimos encontrar el método que mejor se ajuste a sus capacidades. En esta búsqueda, creemos que el método seleccionado en el estudio, el del dibujo-conversación, responde de forma adecuada a las expectativas puestas en él: un método de investigación con niños que permita al investigador recoger la opinión y la visión de éstos sobre el proceso de transición educativa.

Este método presenta muchas ventajas, desde su sencillez hasta el control que ofrece al niño sobre la actividad que está realizando. Combinar el dibujo con las conversaciones nos aporta una información rica y directa por parte del niño, que exterioriza de forma libre y amena sus sentimientos, conocimientos, expectativas y necesidades en torno a la transición educativa.

A pesar de la potencialidad que presenta la metodología que estamos implementando y que sucintamente acabamos de presentar, en su desarrollo deberíamos de contemplar algunas potenciales limitaciones que podrían dificultar su adecuado funcionamiento y que tendríamos que considerar. Entre ellas, destacaríamos:

- La aparición de conversaciones espontáneas entre los niños no relacionadas con la temática propuesta y la necesidad consiguiente de tener que reconducir la conversación.

- La existencia de niños introvertidos que participan escasamente en la conversación, que tienden a responder por monosílabos o, en ocasiones, a repetir las respuestas emitidas por el compañero.

- La presencia de niños que intentan agradar al adulto inventando situaciones. Este hecho puede ser aminorado por la presencia de un compañero (pareja en la conversación) que no corrobore su comentario.

- Las situaciones en la que ciertos niños que no se sientan capaces de dibujar lo que se les plantea o que muestren desagrado por la actividad.

- La reproducción del dibujo del compañero ante la falta de ideas propias o, en ocasiones, el refuerzo verbal que el investigador ofrece al otro niño que ha iniciado su dibujo I"Muy bien... me gusta mucho"l y que puede condicionar la imitación por parte del primero.

- La existencia de niños "eco", esto es, aquellos que ante la pregunta del adulto contestan al investigador con la misma pregunta.

Aunque el trabajo en parejas resulta beneficioso para reducir la inseguridad de los niños ante el adulto, hemos comprobado cómo las discusiones de los niños y las ideas que comparten tienen una influencia directa sobre el contenido de sus dibujos. A pesar de que este hecho puede ser entendido como una limitación del método, coincidimos con Punch (2002) al pensar que, en realidad, nos encontramos ante una representación consensuada entre iguales en relación con la realidad que experimentan.

Pensamos que la identificación de todas estas y de otras limitaciones que pudieran surgir durante el proceso de investigación es un requisito de cara a su mitigación o neutralización y, en consecuencia, para la mejora y el desarrollo de esta metodología.

Esperamos que la información que estamos obteniendo del niño, en cuanto que principal protagonista del proceso educativo, pueda repercutir positivamente en el conjunto de éste y, en el caso que 
nos ocupa y preocupa ahora, en la mejora de los procesos de transición entre las etapas de Educación Infantil y Educación Primaria. Será siempre una garantía el escuchar las necesidades reales percibidas y manifestadas por el niño y no sólo lo que pensamos los adultos sobre lo que necesita la infancia.

\section{Consideraciones finales}

Nos encontramos en tiempos de una modernidad líquida (Bauman, 2005), donde el individualismo imperante hace que convivamos con desconocidos con los que no tenemos intención ni necesidad de tratar. La investigación educativa con niños y no sobre niños, supone una respuesta alternativa ante lo que sucede en el macrocontexto en el que nos desenvolvemos, donde "el otro", en nuestro caso el niño, cuestiona con su presencia el conocimiento que tiene el adulto de la infancia y la comprensión recíproca que el "yo" tiene con el "otro".

La posibilidad de escuchar directamente la voz del niño supone afrontar una incertidumbre que ayudará a romper inercias y a tener en cuenta y conocer en profundidad a la infancia.

Entendemos que la investigación con niños puede ser empleada también como un medio para conseguir uno de los retos educativos que Bauman (2007), identifica en nuestro contexto: el de formar ciudadanos capaces de recuperar el espacio público del diálogo y sus derechos democráticos para poder participar en la sociedad y, de esta forma, poder controlar su futuro.

La investigación educativa con niños posibilita el inicio de una nueva concepción de la infancia amparada por la Convención de los Derechos de la Infancia de las Naciones Unidas, (1989) que otorga al niño la posibilidad de ser escuchado y, específicamente en la investigación educativa, el deber por parte del investigador de recoger la perspectiva infantil.

Este hecho no está exento de dificultades y desafíos para el investigador. Así, los dilemas éticos en torno a aspectos relacionados con la investigación, el consentimiento informado, la selección de la muestra, los criterios de exclusión e inclusión configuran algunos de los contenidos más debatidos por parte de los investigadores que afrontan esta compleja tarea.

Nuestro interés se ha centrado no sólo en la exposición de los mismos sino también, en la muestra de un primer estudio exploratorio que recoge la visión infantil sobre la transición educativa de Educación Infantil a Educación Primaria a través de la técnica del dibujo-conversación. Métodos tradicionales e innovadores están al servicio del investigador. No hemos de caer en el reduccionismo de pensar que los métodos nuevos eliminarán el uso de los ya conocidos, en una sobrevaloración del presente frente a tiempos pasados. En realidad, no es tan importante el método a utilizar como el uso que el investigador haga del mismo. La actitud del investigador hacia el método seleccionado puede provocar tanto que un método tradicional se convierta en un método que involucre a la infancia en la investigación educativa, como que un método innovador restrinja la participación infantil.

Poder recoger la visión del niño en relación a temas que le afectan ayuda a tener una visión más completa de la realidad estudiada, pudiendo reducir el sesgo de estudios que recogen en exclusividad la 
perspectiva adulta. Esto implica constatar que el conocimiento acerca de los niños es incompleto si no se tienen en cuenta los conocimientos que los propios niños tienen de si mismos.

Los estudios que recogen la voz del niño no sólo le capacitan en su rol de agente social participativo y competente sino que sus aportaciones sorprenden a los propios investigadores recogiendo experiencias, intereses, preocupaciones y demandas a las que nunca se hubiera podido responder ni comprender, ya que no eran expresadas y si este acto se daba, no eran escuchadas.

A su vez, este hecho abre la posibilidad de poder adecuar diferentes programas educativos, adaptándolos a las necesidades reales percibidas por el niño y no sólo a lo que piensa el adulto que necesita la infancia.

Para finalizar, apuntar tan sólo que nos encontramos ante un ámbito de investigación escasamente desarrollado a nivel internacional, pero que comporta un enorme potencial en la práctica pedagógica, que no puede pasar inadvertido por los investigadores educativos.

\section{Bibliografía}

AlDERSON, Priscilla (1995). Listening to Children: Children, Ethics and Social Research. London: Barnardo's.

ARgos, Javier., CASTRo, Ana., \& EZQUerRA, María del Pilar (2009). "Repensando la práctica pedagógica de nuestras escuelas: algunos vectores potencialmente revilitadores". En Jesús HERNÁNDEZ, SUsana MOLINA, \& María de las Mercedes INDA (EDS.), La escuela hoy. La Teoría de la Educación en el proceso colectivo de construcción del conocimiento. Oviedo: Universidad de Oviedo, 161-171.

BAUMAN, Zygmunt (2007). Los retos de la educación en la modernidad líquida. Barcelona: Gedisa.

(2005). Modernidad líquida. Argentina: Fondo de Cultura Económica.

Borzone, Ana María, \& Rosemberg, Celia Renata (2000). ¿Qué aprenden los niños cuando aprenden a hablar?. El desarrollo lingüístico y cognitivo en los primeros años. Buenos Aires: Aique.

Bourdillon, Michael (2004). "Children in development". Progress in Development Studies, vol. 4, n 2, 99-113.

BROOKS, Margaret (2005). "Drawing as a unique mental development tool for young children: interpersonal and intrapersonal dialogues". Contemporary Issues in Early Childhood, vol.6, n 1, 80-91.

BRUNER, Jerome (1996). The culture of education. Cambridge: MA: Harvard University Press.

BURKE, Catherine (2005). "Play in Focus: Children Researching their Own Spaces and Places for Place". Children, Youth, Environments, vol. 15, $\mathrm{n}^{\circ} 1,27-53$.

Christensen, Pia, \& JAmes, Allison (2000). "Childhood Diversity and Commonality: Some Methodological Insights". En Pia CHRISTENSEN, \& Allison JAMES (EDS.), Research with Children. Perspectives and Practices. London: Falmer Press, 160179

CHRISTENSEN, Pia \& PROUt, Alan (2002). "Working with Ethical Symmetry in Social Research with Children". Childhood, vol. 9, $n^{\circ} 4,477-497$.

CLARK, Alison, \& Moss, Peter (2001). Listening to Young Children: The Mosaic Approach. London: National Children's Bureau and Rowntree Foundation.

(2005) Spaces to play: more listening to young children using the Mosaic approach. London: National Children's Bureau and Rowntree Foundation.

Cocks, Alison (2006). "The ethical maze. Finding an inclusive path towards gaining children's agreement to research participation". Childhood, vol.13, $n^{\circ} 2,247-266$.

CORSARO, William, \& MOLINORI, Luisa (2000). "Entering and observing in children's worlds: a reflection on a longitudinal ethnography of early education in Italy". En Pia CHRISTENSEN, \& Allison 
JAMES (EDS.), Research with children. Perspectives and Practices London: Falmer Press, 179-201.

DalhberG, Gunilla., Moss, Peter., \& PenCE, Alan (1999). Beyond Quality in Early Childhood Education and Care: Postmodern Perspectives. London and Philadelphia : PA: Falmer Press.

DOCKETT, Sue \& PERRY, Bob (1999). "Starting school: what do the children say?" Early Childhood Development and Care, $\mathrm{n}^{\circ}$ $159,107-119$

(2005). "Starting school in Australia is a "bit safer, a lot easier and more relaxing": Issues for parents from culturally and linguistically diverse backgrounds". Early Years, vol. 25, n 3, $271-281$.

DRIESSNACK, Martha (2006). "Draw-and-tell conversations with children about fear". Qualitative Health Research, vol. 16, $n^{\circ}$ $10,1414-1435$

FASOL, Lyn (2003). "Reading Photographs of Young Children: looking at practices". Contemporary Issues in Early Childhood, vol. $4, n^{\circ} 1,32-47$.

FINCH, Sue (1998). An eye for an eye leaves everyone blind: teaching children to settle conflicts without violence. London: National Early Years Network.

Gallacher, Lesley-Anne., \& Gallagher, Michael (2008). "Methodological immaturity in childhood research?. Thinking through "participatory methods" ". Childhood, vol. 15, n 4, 499-516.

JAMES, Allison, \& ProUt, Alan (1990). "Constructing and Reconstructing Childhood". Contemporary Issues in the Sociological Study of Childhood. London : Falmer Press.

LANCASTER, Penny \& Broadbent, Vanessa (2003). Listening to Young Children. Maidenhead: Open University Press.

LEWIS, Ann, \& LINDSAY, Geoff (2000). Researching Children's Perspectives. Buckingham: Open University Press.

LLoYD-SMITH, Mel, \& TARR, Jane (2000). "Researching Children's Perspectives: A sociological dimension". En Ann LEWIS, \& Geoff LINDSEY, Researching Children's Perspectives. Buckingham: Open University Press, 59-70.

MAYALL, Berry (2000). "Conversations with children: working with generational issues". En Pia CHRISTENSEN, \& Allison JAMES, Advocating for children: International Perspectives on Children's Rights. London: Falmer, 120-135.

Miller, Judy (1997). Never too young: how young children can take responsibility and make decisions. London: National Early Years Network/Save the Children.

O'Kane, ClaIRE (2000). "The Development of Participatory Techniques: Facilitating Children's Views about Decisions which Affect Them". En Pia ChrISTENSEN, \& Allison JAMES (EDS.), Research with Children. Perspectives and practices. London: Falmer Press, 160-179.

Powell, Mary Ann, \& SMith, Anne (2009). "Children's Participation Rights in Research". Childhood, vol. 16, nº 1, 124-142.

PRAMLING, Ingrid (2004). "How do children tell us about their childhood". Early Childhood Research and Practice, vol. 6, $n^{\circ} 1$, $1-15$.

PROSSER, Jon (2003). Image - based Research. A Sourcebook for Qualitative Researchers. London \& New York: Routledge Falmer.

Prout, Alan y JAmEs, Allison (1990). "A New Paradigm for the Sociology of Childhood? Provenance, Promise and Problem", en Alan PROUT y Allison JAMES (eds.): Constructing and Reconstructing Childhood. Londres: Falmer, 7-31.

Prout, Alan (2002). "Researching children as social actors: An introduction to the children 5 -16 programme". Children \& Society, vol. 12, nº 1, 60-65.

PuNCH, Samantha (2002). "Research with children. The same or different from research with adults?" Childhood, vol. 9, $n^{\circ}$ 3, 321-341.

RINALDI, Carla (2001). "A pedagogy of listening: a perspective of listening from Reggio Emilia". Children in Europe, vol. 1, 2-5.

Rio, Paola del, SPaggiarI, Sergio y VeCCHI, Vea (2004): Consejos. Las niñas y los niños de $5-6$ años explican a los de 3 la escuela que los acogerá. Barcelona: Octaedro.

RoberTS, Helen (2000). "Listening to Children: and Hearing Them". En Pia CHRISTENSEN, \& Allison JAMES (EDS.), Research with Children: Perspectives and Practices. London: Falmer Press, 225-240

ROBERTSON, Janet (2000). "Drawing: Making thinking visible". En Wendy SCHILLER (Ed.), Thinking through the arts. Amsterdam: Harwood Academic Publishers, 154-162 
SAYeEd, zahirun., \& GUerIN, Ellen. (2000). Early Years Play: A Happy Medium for Assessment and Intervention. London: Fulton.

YOUNG, Lorraine AND BARRETT, Hazel (2001). "Issues of access and identity: adapting research methods with Kampala street children". International Journal of Childhood, vol. 8, n³, 383-395. 